\title{
Allocution de M. Barrillon
}

\author{
PRESIDENT DU COMTŔ TECHNQUE \\ DE LA SOCIÉTÉ HXDROTECHNIQUE DE FRANCE

\section{Speech by M. Barrillon}

Les exposés qui viennent d'être présentés par les éminents Présidents de Sections permanentes sur l'activité de chacun de nos groupes de travail, vous ont, je pense, donné une idée exacte de la multiplicité des travaux auxquels la S.H.F. essaye de donner une impulsion en utilisant l'ardeur et le désintéressement de ses participants.

La même ardeur se retrouve chez les Présidents des diverses Commissions d'études comme celles qui ont accepté de tenir à jour les Cahiers des charges, les Codes d'essais, les méthodes de mesure ou s'occupent de points particuliers d'actualité. A la valeur technique ces Commissions doivent joindre une connaissance des réglements des intérêts particuliers en jeu, des usages de l'étranger, toutes connaissances qui se rencontrent rarement chez la même personne.

Donner la liste de ces travaux n'est pas possible dans le court délai que nous nous sommes imposé. Soyez certains que dans chacun de ces groupes vous rencontreriez la même foi dans l'utilité des efforts accomplis, et cette conviction est la conséquence de ces faits d'expérience, d'une part que chacun trouve à la S.H.F. l'occasion d'exercer une influence plus forte qu'il ne pourrait le faire dans le cadre restreint de son administration ou de son entreprise, d'autre part, que chacun en retire par les échanges de vues et de documentation des enrichissements auxquels il n'aurait pu prétendre dans l'effort isolé.

Les besoins techniques à satisfaire sont grands, la S.H.F. assume la charge d'y contribuer, elle a créé un esprit de la maison que nous voulons et pensons entretenir. Les exposés que vous venez d'entendre en ont apporté la preuve.

$$
\text { *** }
$$

Nous sommes un organe d'étude et de diffusion. Lorsqu'en France on discute sur le manque d'études, sur le manque de moyens de recherches, il est d'usage de prendre comme introduction le chapitre des inventeurs méconnus et d'établir un palmarès posthume des Français qui n'ont pu réussir qu'après s'être fait comprendre à l'étranger. Tout le monde est d'accord que cela est un mal, qu'il faut y porter remede. Le principal remède est de garantir à « celui qui trouve $»$ que ses efforts seront connus, que ses recherches et études seront examinées avec l'esprit bienveillant de juges qui sont eux-mèmes plaideurs, et plaideurs désintéressés.

Le trouveur indépendant dispose à la S.H.F. d'une tribune où exposer ses idées, il sait qu'il y rencontrera des techniciens prêts à l'écouter, a le faire profiter de leur expérience technique ou administrative, prêts à lui signaler les directions nouvelles dans lesquelles il peut s'engager. Tout ceci peut ètre obtenu, grâce à la pleiade de collaborateurs passionnés qui se sentent chez eux à la S.H.F.

Mais ce n'est pas suffisant, il faut encore que chercheurs et trouveurs soient assurés de la large diffusion de leurs idées. Beaucoup y attachent du prix. Sans publications, le zèle des chercheurs risque de se ralentir, cela est mauvais pour la communauté, cela est mauvais pour le chercheur. En effet, le chercheur qui n'aura pas l'espoir de voir ses idées largement diffusées ne rédigera pas; il trouvera plus facile et plus agréable de sauter d'un sujet à un autre, de dissiper son activité créatrice sur des problèmes qu'il se donne lui-mème, et non sur des problèmes utiles pour la technique, sur des problèmes nécessitant une solution dans un temps limité. Dans ces conditions, ses idées ne mûriront pas, elles se disperseront sans fruit, il restera un chercheur.

Si au contraire, le chercheur rédige, il donne aux autres la possibilité de discuter ses idées et d'élargir sa personnalité; mais surtout par le seul fait de la rédaction, il est amené à condenser sa pensée, à trouver les meilleurs moyens d'exposition et de démonstration, à faire une séparation entre ce qui est réellement neuf et original, une distinction entre ce qu'il pouvait seul trou'ver et ce que d'autres auraient aussi bien pu trouver; il est amené à réfléchir sur les raisons et les circonstances qui l'ont amené à trouver. 
(잉 